\title{
ILCEA
}

Revue de l'Institut des langues et cultures

d'Europe, Amérique, Afrique, Asie et Australie

$12 \mid 2010$

La FASP : dix ans après...

\section{Vera Drake et le Times}

L'adstrat juridique cinématographique comme soutien pédagogique du document authentique

\section{Sandrine Chapon}

\section{OpenEdition}

Journals

Édition électronique

URL : http://journals.openedition.org/ilcea/458

DOI : 10.4000/ilcea.458

ISSN : 2101-0609

Éditeur

UGA Éditions/Université Grenoble Alpes

Édition imprimée

ISBN : 978-2-84310-180-9

ISSN : 1639-6073

Référence électronique

Sandrine Chapon, «Vera Drake et le Times », ILCEA [En ligne], 12 | 2010, mis en ligne le 29 septembre

2010, consulté le 02 mai 2019. URL : http://journals.openedition.org/ilcea/458 ; DOI : 10.4000/

ilcea.458

Ce document a été généré automatiquement le 2 mai 2019.

(c) ILCEA 


\title{
Vera Drake et le Times
}

\author{
L'adstrat juridique cinématographique comme soutien pédagogique du \\ document authentique
}

Sandrine Chapon

\section{Introduction}

En 1999, Michel Petit définit la FASP comme genre et postule que «cet ensemble d'ouvrages de fiction présente suffisamment de caractéristiques communes pour justifier une appellation générique, et que la nature de ces caractéristiques peut être résumée par l'appellation de « substrat professionnel » (Petit, 1999, p. 187).

1 Le substrat en question est précieux pour l'enseignant désireux d'utiliser ces ressources dans le cadre de l'enseignement de l'anglais de spécialité et les FASP cinématographique ou télévisuelle sont des supports particulièrement riches puisqu'elles permettent de montrer la relative authenticité d'un milieu professionnel du fait que leurs auteurs sont issus des milieux qu'ils dépeignent. De plus, elles offrent une exposition accrue à une langue authentique et variée qui peut être utilisée à développer les compétences de réception orale et enrichir le lexique spécialisé des apprenants.

2 Néanmoins, didactiser la FASP peut comporter certaines difficultés. On observe en effet, que les auteurs de FASP juridique affectionnent un type de point de vue narratif : c'est l'avocat ou le procureur qui détient le rôle principal et celui-ci réussira à défendre son client ou la société grâce à des talents oratoires hors pair. Sa prestation mettra en lumière l'aspect culturel de la procédure accusatoire du procès anglo-saxon. La difficulté, en situation didactique, peut résider dans la vitesse d'élocution des acteurs qui, même si elle est représentative d'une certaine réalité, n'en est pas moins parfois assez difficile à utiliser pour les tâches de déchiffrage de la chaîne parlée. C'est pour cette raison qu'il peut être aussi intéressant de chercher des documents didactisables dans des fictions qui ne rentrent pas dans la définition de FASP stricto sensu, mais qui offrent des immersions ponctuelles dans des milieux professionnels.

3 Vera Drake, film réalisé par Mike Leigh en 2005, est une fresque sociale dans laquelle l'héroïne mène une double vie d'employée de maison et d'avorteuse clandestine dans le 
Londres d'après-guerre. Cette fiction n'est donc pas, à proprement parler, une FASP puisqu'il s'agit d'un substrat sociologique dans lequel se trouve un adstrat professionnel juridique, une scène de procès en correctionnelle dans laquelle l'héroïne va être traduite en justice. C'est cette séquence qui peut être exploitée à des fins pédagogiques.

4 L'intérêt didactique tient à la nature même de l'œuvre : c'est un film britannique et, s'il y a pléthore de fictions cinématographiques ou télévisuelles américaines, il existe relativement peu de productions anglaises à notre connaissance. L'œuvre s'inscrit dans la grande lignée des films réalistes, ce qui présente plusieurs facettes intéressantes d'un point de vue de la didactique des langues où, comme le remarque Shaeda Isani, le scénario pédagogique utilisant la FASP juridique cherche, entre autre, à « combler les lacunes des étudiants concernant les spécificités culturelles des autres systèmes juridiques comme le droit anglo-saxon » (Isani, 2006, p. 33). Tout d'abord, le film dépeint une certaine époque difficile à imaginer pour les étudiants. Ensuite, il permet de se placer dans une plus grande dimension sociétale. On peut, par exemple, demander en travail en autonomie que les étudiants effectuent des recherches sur les dates de la légalisation de l'avortement en Grande-Bretagne et en France, et le délai limite pour pratiquer une IVG dans ces deux pays. Enfin, l'adstrat est le miroir d'une certaine réalité dans la mesure où il montre une justice moyenne pour des gens moyens. L'avocat commis d'office fait une prestation médiocre et l'accusée, accablée par la situation, peut à peine acquiescer à l'énoncé de son identité.

5 C'est cet adstrat professionnel juridique que nous proposons d'utiliser dans un scénario pédagogique piloté par la démarche actionnelle, conçu pour des étudiants de première année de la faculté de droit de Grenoble. Nous montrerons qu'il peut être intéressant, dans un premier temps, d'utiliser la bande-annonce du film pour contextualiser le scénario et identifier des faits de langues utilisables ultérieurement dans la trame didactique. Nous exposerons comment l'adstrat juridique peut servir ensuite de soutien à la non-fiction, un article du Times, le projet permettant de faire travailler 4 des 5 compétences identifiées par le cadre européen commun de référence pour les langues (CECRL) : la compréhension de l'oral et de l'écrit et la production orale en continu et en interaction.

\section{Bande-annonce : fonction pédagogique}

Nous proposons d'introduire la séquence en étudiant la bande-annonce, dont la traduction anglaise, trailer ou teaser, est beaucoup plus explicite de sa fonction conative comme définie par Jakobson en 1981 que le signifié français. Le Teaser, emprunt au champ lexical du marketing « aguiche », créant ainsi l'envie d'aller voir le film. Il fonctionne sur le déficit d'information (information gap) résultant d'une contradiction sémantique entre deux types de communication non verbale : l'image et la musique.

La bande-annonce a tout d'abord une fonction référentielle puisqu'elle donne une information sur le sujet en brossant un portrait de la vie quotidienne de l'héroïne éponyme, Vera Drake, par une juxtaposition de tableaux succincts. D'un point de vue de la didactique des langues, cette stratégie permet de faire l'économie de la phase de contextualisation. Elle permet aussi, aux apprenants faibles d'accéder immédiatement au sens puisque l'image suffit à la compréhension. La stratégie marketing de la bandeannonce vise à présenter Vera Drake comme un personnage ordinaire, puis à faire le récit de sa vie, de ses pratiques illicites jusqu'au jour de son arrestation. L'intrigue principale 
est révélée dès le départ. L'envie de voir le film est donc alimentée par le désir de découvrir comment cette femme, si discrète et si ordinaire, se fait arrêter. En tronquant le dernier tiers de la bande-annonce, la partie où est dévoilé le secret de Vera, le questionnement du spectateur / étudiant peut être modifié en vue de susciter, à la place, l'envie de savoir ce qu'il va bien pouvoir arriver au personnage principal. En proposant un premier visionnage sans le son, on utilise la fonction expressive de l'image comme élément porteur de sens. Épouse exemplaire et mère attentive, le but visé par le réalisateur est de créer une certaine empathie avec l'héroïne. On propose alors de repasser l'extrait avec le son cette fois. Il y a très peu de paroles et elles servent essentiellement de fonction phatique et viennent corroborer le sentiment porté par l'image. La musique, en revanche crée un contraste avec l'iconographie. Elle remplit une fonction émotive en véhiculant une impression de danger imminent. C'est ce décalage qui est déclencheur de parole. Ici, l'objectif communicationnel est d'anticiper les scènes à venir et de dresser une liste d'hypothèses. La consigne «Imagine what is possibly going to happen » va donner lieu à des productions du type :

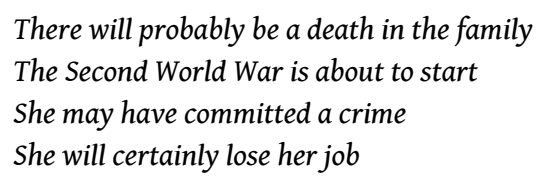

7 La séquence filmée suivante va servir à invalider certaines hypothèses et à en approfondir d'autres. On voit la police arriver au domicile de l'héroïne, interrompre un repas de famille et le visage de Vera se figer dans une expression de consternation. Le langage corporel est renforcé par le court dialogue suivant :

Police officer: We're here to see Mrs Vera Drake.

Vera's husband: What is this about?

Vera: I know why you're her.

Police officer: Why is it that we're here Mrs Drake?

Vera: This is because of what I do.

Police officer: And what is it that you do Mrs Drake?

8 Cet échange verbal permet de confirmer l'hypothèse selon laquelle Vera a enfreint la loi et de ne conserver que les suppositions relatives à celle-ci. On peut attirer l'attention des étudiants sur l'indice offert par Vera Drake, «This is because of what I do ", en leur faisant déduire du temps/aspect du verbe le caractère itératif que revêt le délit de Vera. D'où la production d'énoncés au présent simple du type :

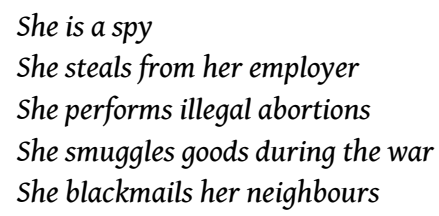

Cette étape crée l'occasion de réactiver en contexte le champ lexical de la qualification des crimes et délits. (Theft, espionage, smuggling, murder, manslaughter, assault, treason, blackmail, arson, etc.)

Nous proposons ensuite une activité visant l'acquisition de la terminologie spécialisée qui va servir de pré-requis pour comprendre la séquence suivante. On attend de cette micro tâche la réalisation par les étudiants, d'un tableau et des productions du type: "If she committed a theft, it's an indictable offense and she faces a two-year time» selon le schéma cidessous : 
Tableau 1 :

Infraction à la loi et conséquences pénales (réalisé collectivement par les étudiants)

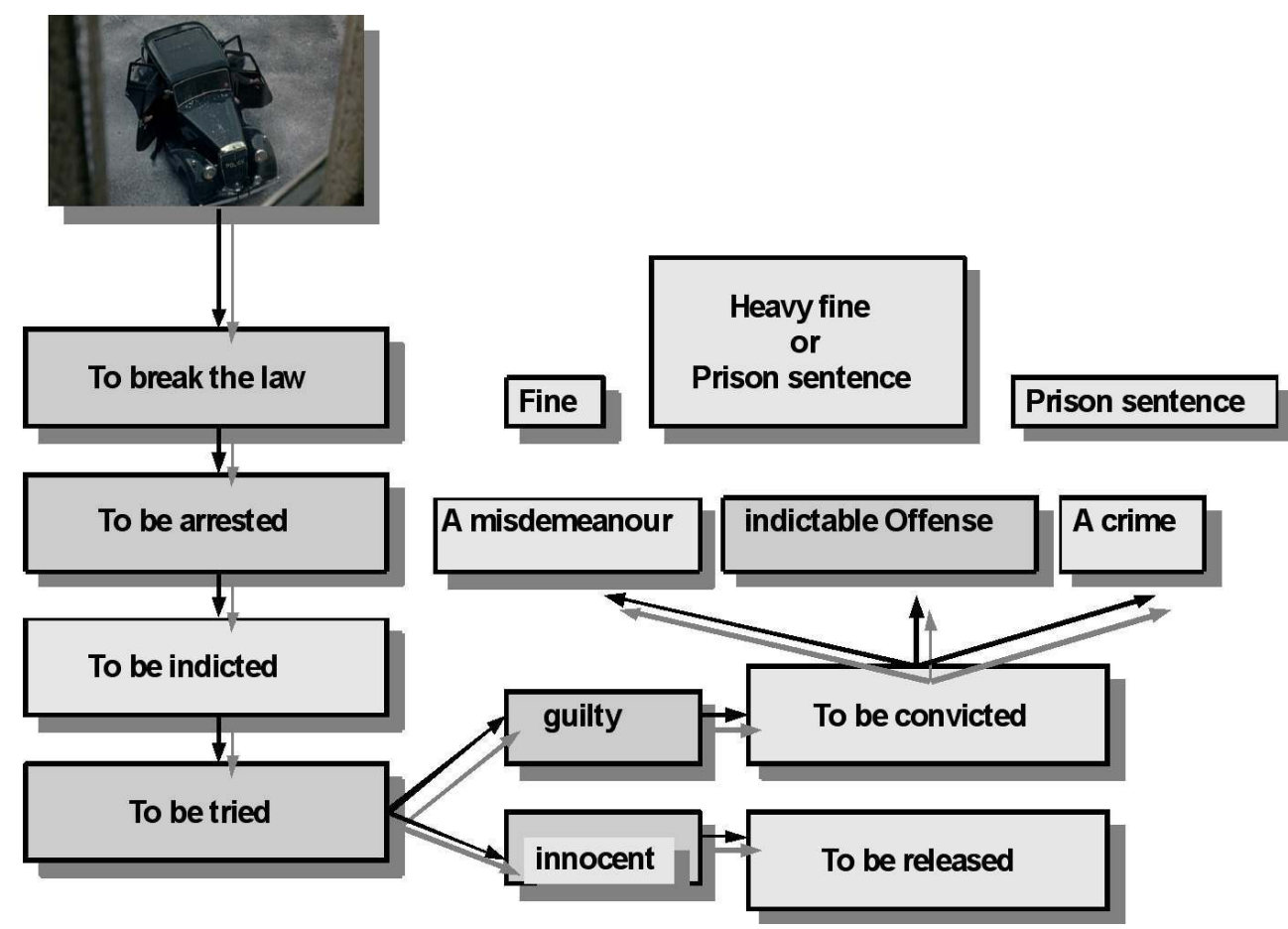

11 La bande-annonce sert donc à mettre en contexte le sujet, à s'approprier le lexique spécialisé et à rebrasser les structures grammaticales nécessaires à la réalisation de la tâche finale. Enfin, elle sert d'activité d'anticipation de la scène du procès de Vera Drake.

\section{Activités de compréhension orale}

L'atout pédagogique de l'adstrat juridique de Vera Drake réside dans la compression temporelle. Le réalisateur choisit de montrer 4 étapes clés du procès: l'énoncé, la reconnaissance des faits, la plaidoirie de l'avocat de la défense et le prononcé du jugement.

12 Cet adstrat permet d'envisager la sémiologie dans toute sa dimension culturelle et comme un fait social global. Premièrement, il donne un aperçu de la sémiotique visuelle : les perruques et les robes des professionnels du droit, les uniformes de police et autres objets sociaux à la signification symbolique forte. Deuxièmement, l'extrait permet de donner un aperçu de la sémiotique kinésique: à savoir l'organisation spatiale des acteurs d'un procès et la gestuelle. Mike Leigh a recours aux plans rapprochés avec la ligne d'horizon à hauteur des spectateurs, ce qui a pour effet de donner l'impression d'être au centre de l'action, d'être partie prenante de la scène. Grâce aux plans en plongée et contre-plongée, on peut amener les étudiants à visualiser l'organisation tridimensionnelle d'un procès en Grande-Bretagne. Le réalisateur a choisi de ne faire aucun plan d'ensemble. C'est par une alternance de gros plans et de plans américains que le procès est filmé. On peut donc proposer aux étudiants d'élaborer le plan d'un tribunal en le comparant au schéma d'un tribunal français. Ceci présente l'avantage didactique d'éviter de décrire une image qui est donnée à tous de voir, ce qui serait une activité quelque peu artificielle. Cette tâche, en revanche, permet un travail de reconstruction et par là même, d'appropriation. 
13 L'adstrat juridique permet aussi d'observer le langage comme produit d'une convention sociale: (Prisoner at the bar, Your Lordship, My Lord) et s'inscrit donc dans la démarche d'acquisition de la langue de spécialité.

14 Enfin, l'énoncé des faits par le greffier permet de valider / invalider les hypothèses établies lors de la phase de mise en contexte que l'on peut proposer sous forme de tâche de discrimination orale des termes juridiques.

Prisoner at the bar, you are charged with using an instrument with intent to procure miscarriage, contrary to section 58 of the Offence Against the Person Act 1861.

The particulars of the offense, are that on the 17th day of November, in the county of London, you did unlawfully use an instrument with intent to procure the miscarriage of a woman named Pamela Mary Barnes. How say you? Are you guilty or not guilty?

Les lexèmes « unlawfully / instrument / offence » permettent aux étudiants d'inférer le chef d'inculpation : to procure the miscarriage ou provoquer des fausses couches et d'anticiper la peine encourue. Cette étape crée une transition naturelle avec l'étude de l'annonce du verdict sous forme de compréhension orale détaillée, une séquence exploitable pour deux raisons :

1. Le discours final du juge a une valeur pédagogique puisque l'accusé doit comprendre sa peine. Le ton est donc didactique et la prosodie est très marquée.

2. Le film étant construit sur l'empathie envers l'accusée, le suspense est créé par la lenteur du phrasé du magistrat.

Ces deux raisons associées rendent les mots porteurs de sens clairement identifiables.

\begin{abstract}
Vera Rose Drake you have committed a crime the gravity of which cannot be overestimated. The Law is very clear and you have wilfully broken that Law. Furthermore in so doing you have put at risk the life of a vulnerable young woman and but for the timely intervention of the medical profession you might have been before me on an even more serious charge than the one that has brought you here today.

Now I have heard your plea of guilty and I've taken that into account and I have listened very carefully to the submissions of your counsel but nothing has been advanced me today on your behalf to persuade me to take any course other than to impose a custodial sentence. Indeed the extreme seriousness of your crime is bound to be reflected in the sentence that I am about to pass and that must serve as a deterrent to others. I therefore sentence you to a term of imprisonment that will be two years and six months.
\end{abstract}

Cet adstrat juridique permet donc d'introduire les savoirs relatifs au lexique juridique de la faute et de la réparation de la faute. Il donne l'occasion aussi de travailler la compétence sociolinguistique : les règles d'adresse et de politesse lors d'un procès, la régulation des rapports entre les statuts ou encore les rituels de fonctionnement d'une communauté linguistique. Enfin, il permet d'aborder la compétence pragmatique relative à la maîtrise du discours des professionnels d'une audience de justice. L'adstrat va pouvoir être utilisé comme document modèle à imiter. On proposera ensuite aux étudiants l'étude d'un texte qui servira entre autre, à déterminer le contexte énonciatif de la tâche finale qu'ils devront accomplir.

\title{
Activité de compréhension écrite : non-fiction
}

Gary Slapper est juriste et écrivain pour le Times. Il relate les procès les plus insolites dans une rubrique intitulée "Weird cases" à la section Law Supplement. L'article sélectionné, The world's weirdest cases, paru le 5 novembre 2007, est composé de vingt paragraphes de quatre lignes en moyenne, chacun décrivant une affaire de justice. Ce document présente 
plusieurs atouts didactiques : premièrement, le caractère léger du sujet constitue une alternative séduisante aux textes primaires utilisés habituellement par les juristes. Ensuite, il vient déconstruire les préjugés selon lesquels les Américains sont les spécialistes des procès farfelus puisque seulement un quart des affaires présentées sont américaines. Enfin, la densité du lexique juridique en fait un document pédagogique de grand intérêt.

In a notorious case heard by Baron Huddleston in November 1884, Captain Thomas Dudley and Edwin Stephens were prosecuted for the murder of a cabin boy, Richard Parker. When the yacht they were sailing from Southampton to Sydney capsized, they found themselves on a dinghy 1,600 miles from shore. After 20 days adrift, they killed Parker, eating his liver and drinking his blood to survive. They were rescued four days later by a German vessel and were convicted of murder at Exeter Assizes, although their death sentences were later commuted to six months imprisonment without hard labour. Their defence of "necessity" was rejected.

A father from Zhengzhou, in China, was refused legal permission to name his son “@” after the keyboard character. Permission was declined on the legal basis that all names must be capable of being translated into Mandarin.

La lecture en continu prenant beaucoup de temps, nous proposons de diviser la classe en groupes de 3. Chaque équipe doit comprendre un seul paragraphe à travers plusieurs activités. Dans un premier temps, l'équipe doit identifier les éléments de l'affaire qu'ils n'ont pas saisis. Cette stratégie permet d'instaurer une activité de communication authentique fondée sur l'information manquante et ceci pour éviter les display questions ou questions purement pédagogiques. Les étudiants doivent trouver eux-mêmes par un questionnement réciproque, les éléments faisant barrage à la compréhension. Cette étape développe la dynamique positive du groupe qui va être nécessaire lors de la réalisation de la tâche finale. Le document permet par ailleurs de constituer un corpus lexical à partir de synonymes ou de définitions proposés par l'enseignant. Cette banque de données servira lors de la réalisation de la tâche finale.

Dans un second temps, nous proposons qu'un porte-parole du groupe relate l'affaire traitée au reste de la classe. La restitution à la classe entière va permettre à chaque étudiant d'avoir une connaissance exhaustive du document tout en constituant un entraînement à la prise de parole en public. Par ailleurs, l'annonce au préalable que la mutualisation des informations va servir à ce que chaque groupe établisse un choix parmi les vingt affaires, permet d'assurer une écoute active de la part de ceux qui ne sont pas en phase de prise de parole en continu.

\section{Activité d'expression orale}

La tâche finale consiste à mettre en scène un des procès décrits dans l'article, en tenant compte des différentes formes de la communication humaine, verbale et non verbale, dans un contexte professionnel.

Chaque groupe est invité à choisir le procès décrit dans l'article de Slapper qui l'inspire le plus, à se répartir les rôles principaux (un juge, un procureur, un avocat de la défense) et à préparer le script selon le modèle culturel et organisationnel proposé dans l'adstrat juridique.

21 La séance suivante, les étudiants ont pour tâche de jouer la scène devant le groupe classe. Le caractère saugrenu des affaires sélectionnées par l'auteur de l'article assure naturellement une écoute active de la part de ceux qui prennent le rôle de spectateurs, et 
le modèle de l'adstrat juridique de Vera Drake fournit un cadre professionnel crédible et permet de mettre en œuvre plusieurs compétences :

- grammaticale (utilisation des temps du passé/lexique du débat contradictoire) ;

- pragmatique (socialisation de la parole en stimulant l'échange autour d'une décision de justice dans une langue professionnelle) ;

- socioprofessionnelle (l'anglais des tribunaux et ses codes).

C'est l'activité de PPI (prise de parole en interaction) qui est visée ici. Cette tâche peut donner lieu à une évaluation somative dont les critères sont les suivants :

- la maîtrise du système phonologique ;

- l'aisance dans la réalisation de la tâche ;

- l'étendue grammaticale ;

- le réinvestissement du lexique juridique ;

- la maîtrise sociolinguistique (peut réagir lors de questions simples ou imprévisibles).

\section{Conclusion}

Le $B O$ hors-série $n^{\circ} 8$ du 30 août 2007 préconise que «chaque séance repose sur des situations et des échanges qui ont du sens pour les élèves, suscitent leur participation active, favorisent les interactions et l'entraide dans le groupe et développent l'écoute mutuelle ». Ce scénario pédagogique, proposé à des étudiants de droit de première année, tente de s'inscrire dans la démarche actionnelle suggérée par le CECRL. Il vise à favoriser la construction collective du sens par chaque étudiant, selon son aptitude de départ, et d'atteindre un niveau d'autonomie langagière dans une situation contrôlée. Le choix d'utiliser un extrait court d'une fiction cinématographique, de répartir le travail de déchiffrage global du texte écrit et de demander aux étudiants de finir de préparer la tâche finale chez eux, répond à la nécessité de mettre à profit le temps de face à face pédagogique pour privilégier les compétences d'expression orale. En effet, les étudiants en droit de la faculté de Grenoble ayant à ce jour 24 heures de langue prévues par leur règlement d'étude pour chaque année de licence, il nous est apparu indispensable de viser en priorité les compétences qui sont très certainement celles que l'étudiant a le moins la possibilité de pratiquer en dehors d'un cours d'anglais.

En procédant à une opération de transcodage où il passe de l'activité de réception à celle de production, l'étudiant est mis en situation d'acteur avec des tâches à accomplir. La démarche vise à consolider les éléments linguistiques et pragmatiques nécessaires à la construction de la compétence de communication. Les étudiants visés n'ayant bien souvent qu'un niveau d'utilisateur élémentaire selon l'échelle de compétences en langue des niveaux communs de référence, ce scénario cherche à développer les aptitudes linguistiques dans le contexte professionnel juridique. 


\section{BIBLIOGRAPHIE}

Bulletin officiel de l'Education nationale, 2005, [En ligne], Décret n 2005-1011 du 22 août 2005 relatif à l'organisation de l'enseignement des langues vivantes étrangères dans l'enseignement scolaire, à la réglementation applicable à certains diplômes nationaux et à la commission académique sur l'enseignement des langues vivantes étrangères, consulté le 07/09/2009.

Bulletin officiel de l'Education nationale, 2007, [En ligne], Hors série $n^{\circ} 8$ du 30 août. Mise en œuvre du cadre européen commun de référence pour les langues. Mise en œuvre du socle commun de connaissances et de compétences, consulté le 01/10/2009.

Conseil de l'Europe, 2009, [En ligne], Manuel pour relier les examens de langues au Cadre Européen commun de référence pour les langues, consulté le 17/01/2010.

FRIES-VERDEIL, Marie-Hélène, « Mise en cohérence de l'anglais de spécialité et du CECRL en France : difficultés et enjeux », Asp, n56, 2009, p. 105-119.

ISANI, Shaeda, « Revisiting cinematic FASP and English for legal purposes in a self-learning environment ». Les Cahiers de l'APLIUT « Cinéma et Langue de spécialité », n² 25/1, 2006, p. 26-38. ISANI, Shaeda, «Specialised fictional narrative and lay readership : Bridging the accessibility gap », Asp, n 56, 2009, p. 45-65.

ISANI, Shaeda, «Popular films as didactic supports in ESP teaching - selection criteria and ethical considerations », Aspect de la fiction à substrat professionnel,M. Petit (dir.), coll. « Travaux 20.25 », 2004, p. 121-132.

JACKOBSON, Roman, Éléments de linguistique générale, vol. I et II, Paris, Minuit. 1981.

JuLIÉ, Kathleen, Enseigner l'anglais, Paris, Hachette Education, 1994.

MARTINEZ, Pierre, La didactique des langues étrangères, Paris, PUF, 1996.

MONNATEUIL, François, [En ligne], « Approche actionnelle et enseignement des langues vivantes dans la voie professionnelle », consulté le 11/06/2009, 2009.

PETIT, Michel, « La fiction à substrat professionnel : une autre voie d'accès à l'anglais de spécialité », Asp, n²3/26, p. 57-81, 1999.

TAHHAN, Brigitte, [En ligne], « Programme de langue vivante dans la voie professionnelle », consulté le 15/03/2010, 2009.

\section{Corpus}

SLAPPER, Gary, 2009, Weird Cases: Comic and bizarre Cases from courtrooms around the world. Wildy, Simmons and Hill Publishing.

EIGH, Mike, Vera Drake, 2005.

\section{RÉSUMÉS}

Cet article propose de décrire les activités d'un scénario pédagogique, réalisé pour des étudiants de première année de droit de la faculté de Grenoble dans le cadre de l'apprentissage de l'anglais 
de spécialité. L'adstrat professionnel juridique que l'on trouve dans le film Vera Drake peut servir de support dans une démarche de pédagogie de projet qui vise l'authenticité de tâches menées par les étudiants, à savoir la mise en scène d'une étape d'un procès au pénal selon le modèle de l'adstrat professionnel. L'article montrera comment le projet, piloté par l'objectif d'appropriation de la langue et culture de spécialité, utilise dans un second temps le document authentique, un article du Times, puisé dans la rubrique « law supplement », comme outil lexical et de création de contexte énonciatif pour réaliser la tâche finale.

This article proposes to portray the different steps of a learning scenario created for students enrolled in the first year of the Grenoble law school who chose English as a foreign language as part of their curriculum. The court room extract that can be found in the film Vera Drake may be used as resource material to be copied according to the pedagogic strategy which aims at creating authentic tasks for the learner in English for Specific Purposes (ESP). The students are required to perform a task coherent with their professional goal, that is to say, they will be expected to act a prominent scene from a criminal trial. This article will describe how the scenario is led by the necessity to perform meaningful tasks using the target language and also uses an article taken from the Times, law supplement, as a "legal words" tool box and inspiration to create the denunciative context necessary to perform the play.

\section{INDEX}

Keywords : cinematographic fiction, court-room drama extract, CEFRL, English for Specific purposes (ESP), legal English, task based language learning (TBLL)

Mots-clés : fiction cinématographique, adstrat professionnel juridique, CECRL, langue de spécialité, anglais juridique, démarche actionnelle 